\title{
Multimodality Assessment of Cardiac Involvement in Churg-Strauss Syndrome Patients in Clinical Remission
}

\author{
Wojciech Szczeklik, MD, PhD; Tomasz Miszalski-Jamka, MD, PhD; Lucyna Mastalerz, MD, PhD; \\ Barbara Sokolowska, MD; Jerzy Dropinski, MD, PhD; Robert Banys, BSc; \\ Kan N Hor, MD; Wojciech Mazur, MD; Jacek Musial, MD, PhD
}

\begin{abstract}
Background: Cardiac involvement in Churg-Strauss syndrome (CSS) is not uncommon, but its frequency varies widely and may depend on the activity of the disease. Therefore, the cardiac involvement in CSS patients in clinical remission was assessed in the present study.

Methods and Results: In 20 CSS patients in remission and 20 sex- and age-matched healthy controls, an ECG stress test, echocardiography, and 24-h ECG Holter monitoring were performed, together with cardiac magnetic resonance imaging (CMRI). Cardiac involvement was present in 90\% (18/20) of CSS patients. Left ventricular ejection fraction (LVEF) was on average lower in the CSS group than in controls $(P<0.05)$, with 7 patients showing systolic heart failure (LVEF $<50 \%)$. cMRI changes included late gadolinium enhancement lesions in the LV in $89 \%$ of patients (17/19), present in all layers of the myocardium. Signs of ongoing inflammation (early gadolinium enhancement) and edema (T2-weighted imaging) were present in 6/19 patients. Holter monitoring revealed both supraventricular and ventricular arrhythmias more frequently in CSS patients when compared with controls $(P<0.05)$. Absolute eosinophil count before the initiation of treatment was higher in rhythm disturbances $(P<0.05)$, and inversely correlated with LV systolic function (rho -0.65).
\end{abstract}

Conclusions: Heart involvement in CSS patients who are in clinical remission is very common. It is characterized not only by fibrosis, but also by an active inflammatory process. The latter finding might influence therapeutic decisions in CSS patients in full clinical remission. (Circ J 2011; 75: 649-655)

Key Words: Cardiac MRI; Churg-Strauss syndrome; Remission; Vasculitis

$\mathbf{C}$ hurg-Strauss syndrome (CSS) is a rare, systemic, necrotizing small-vessel vasculitis, characterized by eosinophil infiltration of various tissues and bronchial asthma. ${ }^{1,2}$ Cardiac involvement in CSS is not uncommon; depending on the report, its frequency varies widely $(16-92 \%){ }^{3,4}$ It has been suggested that cardiac changes are associated with a poor prognosis and high mortality, if left untreated. ${ }^{5}$ Two possible mechanisms of cardiac involvement in CSS have been postulated: vasculitis-related ischemia ${ }^{6}$ and eosinophilic infiltration of the myocardium. ${ }^{7}$ However, the former is being questioned, because cardiac involvement is less common in the antineutrophil cytoplasmic antibody (ANCA)-positive patients. ${ }^{8} \mathrm{~A}$ variety of clinical presentations have been described in this patient population: myocarditis with cardiomyopathy, ${ }^{9}$ pericarditis $(25 \%)$, pericardial effusion (up to $22 \%$ of patients), heart failure (18\%), ventricular and supraventricular arrhythmias, and sudden cardiac death.,8,80

Differences reported in both frequency and the character of heart involvement may be partly explained by the different examination techniques used. Although early reports were mainly based on clinical and autopsy findings, the more recent ones utilized both echocardiography ${ }^{11,12}$ and cardiac magnetic resonance imaging (cMRI). ${ }^{13-18}$ These differences may also depend on the activity of the disease.

We decided to use a multimodality approach to assess cardiac involvement in a group of CSS patients in clinical remission, including ECG (with 24-h Holter monitoring), echocardiography and cMRI.

Received August 4, 2010; revised manuscript received September 30, 2010; accepted October 28, 2010; released online December 3, 2010 Time for primary review: 35 days

Department of Medicine, Jagiellonian University Medical College, Krakow (W.S., T.M.-J., L.M., B.S., J.D., J.M.); Center for Diagnosis, Prevention and Telemedicine, John Paul II Hospital, Krakow (T.M.-J., R.B.), Poland; Cincinnati Children's Medical Center, Cincinnati, OH (K.N.H.); and The Heart and Vascular Center at The Christ Hospitals, Cincinnati, OH (W.M.), USA

Funding: This project was supported by the grant from Polish Ministry of Science and Higher Education, NR R13 00102.

Mailing address: Wojciech Szczeklik, MD, PhD, Department of Medicine, Jagiellonian University Medical College, Skawińska 8, 31-066

Kraków, Poland. E-mail: wszczeklik@gmail.com

ISSN-1346-9843 doi:10.1253/circj.CJ-10-0772

All rights are reserved to the Japanese Circulation Society. For permissions, please e-mail: cj@j-circ.or.jp 


\section{Methods}

\section{Subjects}

The study group comprised 20 consecutive patients (13 women, 7 men; aged 42.9 \pm 9.2 years) diagnosed with CSS (based on the American College of Rheumatology (ACR) 1990 criteria, ${ }^{19}$ and 20 sex- and age-matched healthy controls. In total, 5 patients fulfilled 4 of the ACR criteria, 12 fulfilled 5 and 3 fulfilled all of the 6 criteria. All patients had a history of allergic rhinitis and asthma with elevated peripheral blood eosinophilia ( $>10 \%$, before the initiation of treatment). The patients had a documented history of neuropathy (11 patients: 55\%), pulmonary infiltrates (18: 90\%), paranasal sinus abnormalities (19: 95\%) and extravascular eosinophil infiltration on tissue biopsy (10: 50\%). Only in 5 patients $(25 \%)$ was a positive perinuclear pattern of ANCA detected on repeated examinations.

The Birmingham Vasculitis Activity Score (BVAS $)^{20}$ served to assess disease activity and only patients in remission (BVAS $\leq 1)$ were studied.

All participants gave informed consent to participate. The study protocol complied with the Helsinki Declaration and was approved by the Jagiellonian University Ethics Committee.

\section{Study Protocol}

In all patients and controls we conducted basic laboratory tests (with plasma cardiac enzymes and levels of N-terminal brain natriuretic peptide (NTpro-BNP)), echocardiography, and 24-h ECG Holter monitoring. All the controls and 19 patients with CSS underwent cMRI $(n=20) ; 1$ of the patients was excluded because of claustrophobia. A graded exercise stress test (GXT) was performed by all 20 controls and 18 CSS patients; 2 patients were excluded because they were unable to exercise (class IIIb CHF, severe neuropathy).

24-h ECG Holter Monitoring A 3-channel digital recorder (Aspel, HolCARD $24 \mathrm{~W}$, Poland) was used and data were subsequently analyzed using software provided by the manufacturer. Hour heart rate (HR), number of ventricular (VEs) and supraventricular extrasystoles (SVEs) were analyzed.

GXT Resting HR and blood pressure (BP) were measured and a 12-lead ECG was recorded before exercise. All patients underwent a treadmill exercise, according to a standard Bruce protocol. Each test was terminated in accordance with the AHA/ACC guidelines. Test results were defined as positive using standard criteria. ${ }^{21}$

Cardiac MRI Breath-hold, ECG-gated cMRI was performed with a 1.5-T whole-body scanner (Magnetom Sonata Maestro Class, Siemens, Erlangen, Germany) equipped with dedicated cardiac software and 6-element cardiac phased array coil (sandwich type). After scout imaging, cine and morphologic imaging was performed in the short-axis as well as in 2-chamber, 4-chamber and long-axis apical views. Cine imaging was obtained by steady-state free precession gradient echo technique (slice thickness $8 \mathrm{~mm}$, no gap, TR/TE 40/1.2 ms, flip angle $59^{\circ}$ ). Morphologic images were acquired with the body coil using TSE T2-weighted imaging (slice thickness $8 \mathrm{~mm}$, no gap, TR depending upon RR interval, TE $78 \mathrm{~ms}$, flip angle $180^{\circ}$ ) and TSE T1-weighted imaging (slice thickness $8 \mathrm{~mm}$, no gap, TR depending upon RR interval, TE $6.9 \mathrm{~ms}$, flip angle $180^{\circ}$ ). TSE T1-weighted imaging was performed before and immediately after intravenous infusion of $0.15 \mathrm{mmol} / \mathrm{kg}$ body weight gadobutrol (Gadovist, Bayer Schering Pharma, Berlin, Germany). At 10 min after contrast injection late gadolinium-enhanced (LGE) imaging was per- formed using a T1-weighted segmented inversion-recovery pulse sequence (slice thickness $8 \mathrm{~mm}$, no gap, inversion time set to null normal myocardium).

Cardiac MRI Analysis TSE T1-weighted pre- and aftercontrast, TSE T2-weighted, cine and LGE images were assessed off-line by 2 independent observers who were unaware of the clinical data. In case of discrepancy in qualitative assessment consensus was reached. A 17-segment model of the left ventricle (LV) was used for analysis.

Cine Images Short-axis as well as 2-chamber, 4-chamber and long-axis apical views were used for segmental wall motion analysis. Segmental myocardial contractile function was assessed in terms of endocardial motion and/or systolic wall thickening as 1, normal; 2, hypokinetic; 3, akinetic; 4, dyskinetic and expressed as a wall motion score index. Endocardial and epicardial borders were outlined on the short-axis images to calculate both left and right ventricular (RV) end-diastolic volumes (EDV), end-systolic volumes (ESV), myocardial mass and ejection fraction (EF). EDV, ESV and myocardial mass were indexed to body surface area. LV systolic dysfunction was defined as LVEF $<50 \%$. Cine shortaxis midventricular images were used for global circumferential strain $\left(\varepsilon_{c c}\right)$ measurement as described previously. ${ }^{22}$ The assessment was performed using the TomTec Diogenes ${ }^{\circledR}$ software (TomTec Imaging Systems, Munich, Germany), which is a vector-based analysis tool, intended for the quantification of myocardial deformation and movement using dynamic 2-dimensional cMRI data. Diogenes ${ }^{\circledR}$ magnetic resonance feature tracking takes advantage of an approach whereby features are not "detected", rather they are "tracked"; that is, followed in time, starting from a reliable existing instantaneous trace that is manually drawn by the operator over a single frame.

TSE T1- and T2-Weighted Images By quantitative analysis the mean T2 and mean T1 signal intensities (SI) (pre- and post-contrast) were measured on the short-axis view of individual LV segments, in the region of interest encompassing the LV myocardium on the mid-LV short-axis view as well as in skeletal muscle (erector spinae or latisimus dorsi) in the same slice. The T2 myocardial SI was related to the skeletal muscle SI and a T2-SI ratio $\geq 2.0$ in at least $1 \mathrm{LV}$ segment was considered to reflect abnormal water accumulation. The myocardial early gadolinium enhancement (EGE) ratio was calculated as the enhancement of myocardium divided by the enhancement of skeletal muscle. ${ }^{23}$ An EGE ratio $\geq 4$ in at least $1 \mathrm{LV}$ segment was considered abnormal.

LGE Images LGE images were assessed qualitatively for the presence and location of hyperintense lesions in contrast to hypointense viable myocardium. The number of affected segments was calculated and RV involvement was determined. LGE lesions were defined as transmural, subendocardial (adjacent to endocardium), midwall and subepicardial (adjacent to epicardium). Figure shows the cMRI findings in a 58-year-old woman with CSS.

Transthoracic Echocardiography (TTE) TTE was performed in the parasternal long-axis, short-axis and apical 4-chamber, 2-chamber and long-axis views with a Vivid 7 ultrasound system (GE Vingmed Ultrasound A/S, Horten, Norway). The analysis was performed off-line by an independent, experienced viewer who was unaware of the clinical and cMRI data. LVEF, LVEDV and LVESV were calculated. Systolic dysfunction was defined as LVEF $<50 \%$. LV diastolic dysfunction and the severity of valve dysfunction were evaluated in accordance with the guidelines published by the American Society of Echocardiography. ${ }^{24,25}$ 


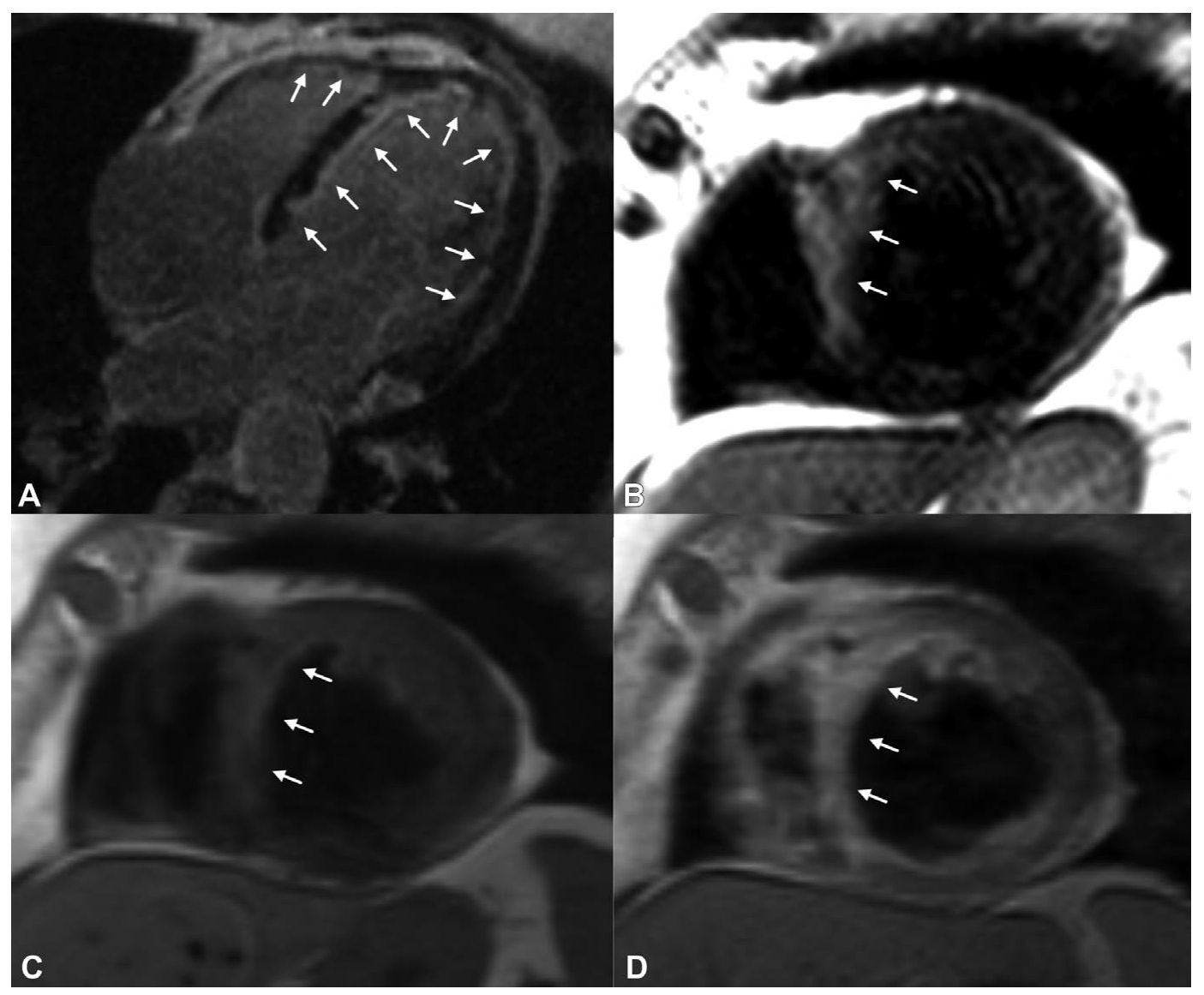

Figure. Late gadolinium enhancement (LGE) image (A) in the 4-chamber apical view, as well as TSE T2-weighted (B), pre- (C) and after-contrast TSE T1-weighted (D) images in the short-axis left ventricular view in a 58-year-old woman with Churg-Strauss syndrome. LGE image shows extensive subendocardial fibrosis in the left and right ventricles (arrows: A). Apical septal segment demonstrates both increased T2 signal intensity (arrows) on the TSE T2-weighted image (B), suggesting myocardial edema, and early gadolinium enhancement (arrows) on the after-contrast TSE T1-weighted image (D), reflecting increased myocardial hyperemia/capillary leak. Cardiac magnetic resonance imaging revealed mild left ventricular systolic dysfunction (ejection fraction $44 \%$, apical akinesis), which was corroborated by transthoracic echocardiography (TTE). Mitral inflow profile registered by pulse-wave Doppler during TTE revealed impaired diastolic dysfunction with $E / A=0.66$ and deceleration time $380 \mathrm{~ms}$.

\section{Statistical Analysis}

Statistical analysis was performed using StatSoft, Inc (2008) STATISTICA software, version 8.0. (www.statsoft.com). Data were checked for normality and are presented as the mean \pm SD, mean $(95 \%$ confidence interval) or median $(25-$ $75 \%$ percentile), where applicable. The comparison between group means was made using Student's t-test, after ascertaining normal distribution. For comparison of not normally distributed data, the Mann-Whitney U-test was used and for categorical variables, the chi-square test and Fisher's exact test. Correlation was tested using the Spearman rank test. Organ involvement was calculated as percentages of affected individuals. $\mathrm{P}$ values less than 0.05 were considered statistically significant.

\section{Results}

In total, 8 patients had complained either in the past or recently of chest pain, arrhythmia or dyspnea on exertion. Based on the hospital charts 5 CSS patients suffered from pericarditis, 8 from myocarditis and 1 met WHO criteria for myo- cardial infarction in the presence of normal coronary anatomy demonstrated by invasive coronary angiography performed within the first $24 \mathrm{~h}$ of the beginning of chest pain. Two patients suffered a witnessed sudden cardiac death and were successfully resuscitated and in one of them a large intraventricular thrombus in the LV was detected and it dissolved following anticoagulant treatment. At the time of examination 2 patients were in NYHA class III, and 4 in class I. In neither group were current smokers found; a history of smoking was given by 3 patients and 4 controls; 4 patients in the CSS group had hypertension. None had diabetes. One subject in the control group had hypertension. At the time of study all patients were on oral corticosteroids (median dose $8 \mathrm{mg}$; range 6$16 \mathrm{mg}$ of methylprednisolone); 9 received additional immunosuppressive therapy according to the current guidelines. Of the CSS group, $7(35 \%)$ were on angiotensin-converting enzyme inhibitors (ACEI), 5 were on $\beta$-blockers (25\%), 5 on diuretics (25\%), 2 on aspirin (10\%) and 2 on statins (10\%). None of the controls subjects took any medication.

The demographic and clinical characteristics are presented in Table 1. Peripheral blood eosinophilia was higher 


\begin{tabular}{|c|c|c|}
\hline & CSS $(n=20)$ & Controls $(n=20)$ \\
\hline $\operatorname{Sex}(F / M)$ & $13 / 7$ & $13 / 7$ \\
\hline Age, years & $42.9 \pm 9.2$ & $43 \pm 10.34$ \\
\hline $\mathrm{BMI}, \mathrm{kg} / \mathrm{m}^{2 *}$ & $24.1 \pm 3.85$ & $23.2 \pm 3.3$ \\
\hline Blood eosinophilia, cells $/\left.\mu\right|^{\star *}$ & $261(126-437)$ & $103(71-212)^{\dagger}$ \\
\hline p-ANCA, n (\%) & $5(25)$ & $0^{+}$ \\
\hline Total cholesterol, $\mathrm{mmol} / \mathrm{L}^{*}$ & $5.0 \pm 0.8$ & $4.9 \pm 0.7$ \\
\hline LDL-C, mmol/L* & $2.8 \pm 0.6$ & $2.7 \pm 0.8$ \\
\hline $\mathrm{HDL}-\mathrm{C}, \mathrm{mmol} / \mathrm{L}^{*}$ & $1.8 \pm 0.4$ & $1.7 \pm 0.4$ \\
\hline Triglycerides, $\mathrm{mmol} / \mathrm{L}^{*}$ & $1.2 \pm 0.5$ & $1.2 \pm 0.5$ \\
\hline Abnormal CRP & 1 & 0 \\
\hline Plasma fibrinogen, $g / L^{*}$ & $3.7 \pm 1.2$ & $3.2 \pm 0.6$ \\
\hline Creatinine, $\mu \mathrm{mol} / \mathrm{L}^{*}$ & $74.9 \pm 12$ & $80.9 \pm 12.3$ \\
\hline NT-pro BNP ${ }^{* *}(\mathrm{NR}<450 \mathrm{pg} / \mathrm{ml})$ & $40(20-74.5)$ & $20(20-20)^{\dagger}$ \\
\hline Abnormal troponin I & 0 & 0 \\
\hline
\end{tabular}

${ }^{\dagger} \mathrm{P}<0.05$. ${ }^{*}$ Mean value \pm standard deviation. ${ }^{* *}$ Median $[25-75$ interquartile range].

CSS, Churg-Strauss syndrome; BMI, body mass index; p-ANCA, antineutrophil cytoplasmic antibodies, perinuclear pattern; LDL-C, low-density lipoprotein cholesterol; HDL-C, high-density lipoprotein cholesterol; CRP, C-reactive protein; NT-proBNP, N-terminal brain natriuretic peptide; NR, normal range.

in the CSS group than in the controls $(\mathrm{P}<0.05)$. Patients with systolic heart failure had higher eosinophilia than the remaining patients, both before implementation of corticosteroids $(8,000$ cells/ $\mu 1$ [range 5,000-9,000] vs. 5,000 [2,200-8,000], respectively; $\mathrm{P}<0.05$; correlation between eosinophilia and LVEF rho $-0.65 ; \mathrm{P}<0.05)$ and at the time of study (454 [range
222-587] vs. 176 [109-268], P<0.05). Serum NT-proBNP levels were increased in the CSS group when compared with the controls $(\mathrm{P}<0.05)$; however, this difference was caused by 2 CSS patients, both with symptomatic LV failure (NYHA III class). In the others NT-proBNP levels were within the normal range.

\section{GXT Results}

No ECG changes characteristic of ischemia at rest were recorded in either patients or controls. Partial right bundle branch block was identified in 3 patients and left posterior fascicular block in 2 . One patient presented with ectopic atrial rhythm. All these patients were asymptomatic from the cardiac perspective.

GXT was positive in 4 CSS patients and none of the controls $(\mathrm{P}<0.05)$. In neither group were angina or equivalent symptoms noted during the test nor in the recovery period; BP and HR changes were similar. Patients in the CSS group achieved a lower exercise workload than the control group (12.3 \pm 2.2 vs. $14.6 \pm 1.8$ metabolic equivalents, respectively; $\mathrm{P}<0.001)$. In 4 patients with a positive stress test the peak eosinophilia before initiation of treatment was significantly higher than in the remainder $(11,000$ cells/ $\mu 1[8,750-21,000]$ vs. 3,784 [1,800-7,000], respectively; $\mathrm{P}<0.01)$.

\section{4-h Holter Monitoring}

No differences in HR, HR variability, episodes of bradycardia or pauses $>2 \mathrm{~s}$ were observed between the CSS and control groups. Several different types of arrhythmias were documented in the CSS patients: VEs abnormal for age in 5 patients (including VE bigeminy, trigeminy, pairs and ventricular tachycardia) accompanied by abnormal SVEs in 4 of

Table 2. Findings of cMRI of the Study Groups

cMRI parameters

LVEF (\%)

LVEDV index $\left(\mathrm{ml} / \mathrm{m}^{2}\right)$

LVESV index $\left(\mathrm{ml} / \mathrm{m}^{2}\right)$

LVMass index $\left(\mathrm{g} / \mathrm{m}^{2}\right)$

$\varepsilon_{\mathrm{cc}}(\%)$

RVEF (\%)

RVEDV index $\left(\mathrm{ml} / \mathrm{m}^{2}\right)$

RVESV index $\left(\mathrm{ml} / \mathrm{m}^{2}\right)$

RVMass index $\left(\mathrm{g} / \mathrm{m}^{2}\right)$

Myocardial SI-T2 ratio at mid LV level

Myocardial EGE ratio at mid LV level

Increased myocardial SI-T2 and/or EGE ratio

Pericardial effusion

LV LGE

LV LGE: no. of segments

Subepicardial LGE

Midwall LGE

Subendocardial LGE

Extensive (>10 seg.) subendocardial LGE

RV LGE

\begin{tabular}{|c|c|c|c|c|c|}
\hline \multicolumn{5}{|c|}{ CSS patients } & \multirow{2}{*}{$\begin{array}{c}\text { Controls } \\
\begin{array}{c}\text { All } \\
\text { subjects } \\
(n=20)\end{array}\end{array}$} \\
\hline $\begin{array}{l}\text { LVEF } \\
<50 \% \\
(n=6)\end{array}$ & $\begin{array}{l}\text { LVEF } \\
\geq 50 \% \\
(n=13)\end{array}$ & $\begin{array}{c}\text { Impaired } \\
\text { diastolic } \\
\text { function }(n=7)\end{array}$ & $\begin{array}{c}\text { Normal } \\
\text { diastolic } \\
\text { function }(n=12)\end{array}$ & $\begin{array}{c}\text { All } \\
\text { patients } \\
(n=19)\end{array}$ & \\
\hline $38.8 \pm 14.3$ & $66.1 \pm 8.8^{\dagger}$ & $44.3 \pm 17.9$ & $65.2 \pm 10.1^{\ddagger}$ & $57.5 \pm 16.7$ & $61.4 \pm 2.9$ \\
\hline $91.5 \pm 35.5$ & $68.2 \pm 10.7^{*}$ & $90.6 \pm 32.0$ & $66.8 \pm 10.7^{\ddagger}$ & $75.6 \pm 23.5$ & $74.3 \pm 11.0$ \\
\hline $60.0 \pm 37.8$ & $23.4 \pm 8.7^{\star}$ & $54.6 \pm 37.3$ & $23.5 \pm 9.3^{\ddagger}$ & $35.0 \pm 27.5$ & $28.7 \pm 5.0$ \\
\hline $66.0 \pm 15.4$ & $57.3 \pm 12.5$ & $64.2 \pm 14.9$ & $57.6 \pm 12.9$ & $60.1 \pm 13.7$ & $52.8 \pm 9.6$ \\
\hline$-13.0 \pm 5.7$ & $-21.5 \pm 4.4^{*}$ & $-14.3 \pm 6.1$ & $-21.5 \pm 4.7^{\star}$ & $-18.8 \pm 6.2$ & $-22.0 \pm 2.4^{*}$ \\
\hline $58.9 \pm 9.7$ & $61.9 \pm 6.9$ & $61.0 \pm 6.0$ & $61.0 \pm 8.8$ & $61.0 \pm 7.8$ & $58.1 \pm 6.5$ \\
\hline $56.6 \pm 9.5$ & $69.4 \pm 12.7^{*}$ & $60.3 \pm 12.8$ & $68.4 \pm 10.7$ & $65.4 \pm 13.0$ & $76.6 \pm 11.8^{\#}$ \\
\hline $23.6 \pm 8.2$ & $26.7 \pm 7.9$ & $23.7 \pm 8.0$ & $26.9 \pm 8.0$ & $25.7 \pm 7.9$ & $32.2 \pm 7.6^{\#}$ \\
\hline $16.3 \pm 3.1$ & $18.0 \pm 3.9$ & $16.0 \pm 3.1$ & $18.3 \pm 3.8$ & $17.5 \pm 3.7$ & $18.6 \pm 3.8$ \\
\hline $1.4 \pm 1.1$ & $0.6 \pm 0.4^{*}$ & $1.4 \pm 1.0$ & $0.6 \pm 0.4^{\ddagger}$ & $0.9 \pm 0.7$ & $0.5 \pm 0.3^{\#}$ \\
\hline $3.5 \pm 1.1$ & $2.1 \pm 1.0^{*}$ & $3.4 \pm 1.4$ & $2.1 \pm 0.7^{\ddagger}$ & $2.6 \pm 1.2$ & $2.2 \pm 0.9$ \\
\hline 5 & $1^{*}$ & 6 & $0 \S$ & 6 & $0^{\#}$ \\
\hline 1 & 0 & 1 & 0 & 1 & 0 \\
\hline 6 & 11 & 7 & 10 & 17 & $1^{* *}$ \\
\hline $10.7 \pm 6.7$ & $2.5 \pm 1.8^{*}$ & $10.3 \pm 5.9$ & $2.0 \pm 1.4 \S$ & $5.1 \pm 5.5$ & $0.05 \pm 0.2^{\star \star}$ \\
\hline 4 & 5 & 4 & 5 & 9 & $1^{\#}$ \\
\hline 4 & 7 & 6 & 5 & 11 & $0^{\star *}$ \\
\hline 5 & $3^{*}$ & 5 & 3 & 8 & $0^{* *}$ \\
\hline 3 & $0^{*}$ & 3 & $0 \ddagger$ & 3 & 0 \\
\hline 5 & 7 & 7 & 5 & 12 & $0^{\star \star}$ \\
\hline
\end{tabular}

${ }^{*} \mathrm{P}<0.05,+\mathrm{P}<0.001$ for LVEF $<50 \%$ vs. LVEF $\geq 50 \%$; $¥ \mathrm{P}<0.05, \$ \mathrm{P}<0.001$ for impaired vs. normal LV diastolic function; \# $<0.05,{ }^{\star *} \mathrm{P}<0.001$ for CSS patients vs. controls.

CMRI, cardiac magnetic resonance imaging; CSS, Churg-Strauss syndrome; LV, left ventricular; EF, ejection fraction; EDV, end-diastolic volume; ESV, end-systolic volume; $\varepsilon$ cc, global circumferential strain; RV, right ventricular; SI, signal intensity; EGE, early gadolinium enhancement; LGE, late gadolinium enhancement; seg, segments. 
these patients. No clinically significant VE or SVE arrhythmias were found in the control group $(\mathrm{P}<0.05)$. In the 5 CSS patients with VEs the peak eosinophilia before initiation of treatment was significantly higher than in the other patients $(9,000$ cells $/ \mu 1$ [range $8,750-11,000$ ] vs. 4,000 [range 1,800 $7,000]$, respectively; $\mathrm{P}<0.05)$. The VEs were more frequent among CSS patients with systolic heart failure than in the rest of the CSS group $(\mathrm{P}<0.05)$. In addition, CSS patients with systolic heart failure had higher mean and minimal HRs in comparison with the other CSS patients (mean HR 86.6 \pm 6.4 vs. $77.5 \pm 8.2$ beats $/ \mathrm{min}$; $\mathrm{min}$. HR $57.3 \pm 8$ vs. $48.9 \pm 5.8$ beats $/ \mathrm{min}$, respectively; both $\mathrm{P}<0.05)$.

\section{Echocardiography}

CSS patients demonstrated overall lower LVEF than controls $(55.9 \pm 15.8 \%$ vs. $63.6 \pm 3.5 \%, \mathrm{P}<0.05)$. Impaired systolic function $(\mathrm{LVEF}<50 \%)$ was present in 7 patients with CSS $(<30 \%$ in 2). When compared with the controls, CSS patients tended to have a higher EDV index $\left(66.2 \pm 24.2\right.$ vs. $55.9 \pm 8.7 \mathrm{ml} / \mathrm{m}^{2}$, $\mathrm{P}=0.08)$ and $\mathrm{ESV}$ index $\left(30.8 \pm 26.4\right.$ vs. $20.7 \pm 5.0 \mathrm{ml} / \mathrm{m}^{2}, \mathrm{P}<$ $0.09)$, but these differences did not reach statistical significance. Diastolic dysfunction was observed in 8 CSS patients (grade I in 4, grade II in 3, and grade III in 1). Of these 8 patients, 6 had an accompanying systolic dysfunction. Diastolic dysfunction (grade I) was also observed in 1 control subject $(\mathrm{P}<0.05)$ suffering from hypertension. Pericardial effusion $(8 \mathrm{~mm})$ was found in 1 patient in the CSS group and in none of the controls. Mitral valve insufficiency was detected in $4 \mathrm{CSS}$ patients (mild in 3 , moderate in 1) and in 1 control (mild). No intraventricular thrombus was found on echocardiographic examination in either group.

\section{Cardiac MRI}

Table 2 summarizes the cMRI findings; 6 CSS patients and none of the controls demonstrated LVEF $<50 \%(\mathrm{P}<0.05)$. In contrast to the TTE data the difference in LVEF between the CSS and control groups did not reach statistical significance, probably because cMRI was not performed in 1 patient with markedly depressed LV function (EF 41\%). Patients with impaired LV systolic function, when compared with those with normal LV systolic function, had decreased $\varepsilon_{\mathrm{cc}}$, higher LVEDV index and LVESV index, as well as a higher myocardial EGE ratio and T2-SI ratio. The same was true for CSS patients with abnormal LV diastolic function, when compared with those who had normal LV diastolic function (Table 2). Both an abnormal T2-SI ratio in at least $1 \mathrm{LV}$ segment suggesting myocardial edema and an EGE ratio reflecting increased myocardial hyperemia/capillary leak were found in 6 patients with abnormal LV diastolic function and in none of the patients with normal LV diastolic function $(\mathrm{P}<0.05)$. Of those 6 patients, 5 demonstrated LVEF $\leq 50 \%$ and in 1 the EF was normal. Pericardial effusion was found in 1 patient and none of the healthy subjects. LGE was present in 17 of $20 \mathrm{CSS}$ patients and in none of the controls. In the CSS patients the total number of LV segments affected by LGE according to localization was: subendocardial, 61; midwall, 38; epicardial, 11 segments. Subendocardial LGE was found more frequently in those with LVEF $\leq 50 \%$ than those with LVEF $>50 \%$ (Table 2). Four patients demonstrated subendocardial LGE lesions on the RV side of the interventricular septum. Twelve patients had LGE lesions located in the free wall of the right ventricle. All of them showed LGE also within LV myocardium. Interestingly, in CSS patients with excessive VEs, as detected by Holter monitoring, the number of segments showing LGE was higher than in those without arrhythmia (11.2 \pm 7 vs. $2.7 \pm 2.4$ segments; $\mathrm{P}<0.05$ ). Patients with an abnormal stress test also had a higher number of segments demonstrating LGE on cMRI than the rest of the CSS group (11.6 \pm 7.6 vs. $2.5 \pm 1.8$ segments, respectively; $\mathrm{P}<0.05)$.

\section{Discussion}

In the present study a multimodality approach to assessing cardiac involvement in CSS patients in remission was used, irrespective of their cardiac complaints and symptoms. This is the first study in this patient population to comprehensive utilize cMRI to assess function (not only by EF but also by $\varepsilon c c)$ as well as characterize the inflammatory process (potentially reversible: EGE, increased T2 signal, and irreversible: LGE). The main finding of our study is that there is a high prevalence of heart involvement and signs of ongoing myocardial inflammation in more than $25 \%$ of CSS patients despite full clinical remission. Surprisingly, many patients with evidence of cardiac involvement by LGE had preserved contractility even when assessed by $\varepsilon$ cc, which is a more sensitive, subtle assessment of contractility than EF alone.

cMRI changes indicative of cardiac involvement were found in 17 of 19 patients (89\%). Additionally, in the 1 patient not examined by cMRI, because of claustrophobia, cardiac abnormalities were documented by echocardiography (EF $41 \%$ with increased LVEDV and accompanying diastolic dysfunction and pulmonary hypertension) (Table S1). LGE changes localized in the LV were by far the most frequent cMRI abnormality, found in as many as $89 \%$ of the CSS patients (17/19), and in none of the healthy subjects. In 12 of those 17, LV LGE was associated with similar changes in the RV. The LGE phenomenon likely reflects irreversible myocardial injury caused by the substitution of viable heart tissue by either necrosis or fibrosis. ${ }^{26,27}$

The patients with a higher number of segments affected by LGE also more often demonstrated a decreased LVEF $(\leq 50 \%)$, positive ECG stress test and ventricular arrhythmias on Holter monitoring as compared with the rest of the CSS group; findings already observed in other cardiomyopathies. ${ }^{28-30}$ These results also indicate that LGE changes (and their location ie, infra) detected in CSS patients in remission carry important clinical consequences.

Previous reports localized LGE changes in CSS patients mostly in the subendocardium, ${ }^{14,15}$ as typically described in Löffler endocarditis. ${ }^{31}$ In contrast, the distribution of the LGE changes in our study was not only in the subendocardium, but also in the intramural and subepicardial myocardium. This difference may be caused partly by patient selection bias. Although the previous studies included mostly patients with otherwise proven cardiac involvement and an active stage of the disease, ${ }^{14,15}$ we analyzed patients in full remission. It is possible then, that changes predominantly located in the subendocardium may identify patients with more severe heart involvement leading to heart failure. Indeed, in our study subendocardial changes were more often associated with impaired LVEF $(<50 \%)$ (Table 2) Of note in CSS patients, myocardial changes outside the subendocardial layer have already been described in case reports and confirmed occasionally by histopathology..$^{13,32}$

Despite the fact that we studied a CSS group in remission, signs of acute ongoing inflammation (eg, edema: increased T2/S1 ratio), hyperemia and capillary leak (increased myocardial EGE) were present in 6 patients and in another were accompanied by pericardial effusion. All these patients had 
either systolic and/or diastolic heart failure. No such changes were documented in the healthy controls or the rest of the CSS group. These abnormalities, usually attributed to acute myocarditis, are potentially reversible. In previous work by Wassmuth et al, they tended to disappear with increased immunosupressive treatment. ${ }^{14}$ As cardiac involvement is one of the main predictors of mortality in CSS, ${ }^{5}$ and an important prognostic factor in the 5 factor score,${ }^{33}$ it is of crucial importance to perform cMRI even in asymptomatic patients with CSS, to search for reversible cardiac pathology. In such patients increasing the dose of immunosuppressive therapy may potentially prevent the development of irreversible fibrosis. Echocardiography and cMRI are complimentary in the comprehensive assessment of cardiac involvement in CSS patients. Although the echocardiographic findings paralleled those of cMRI in the present assessment of EF and chamber dimensions, diastolic dysfunction was detected exclusively by echocardiography. Not surprisingly, only subjects with diastolic dysfunction demonstrated signs of ongoing inflammation, visualized by cMRI. It might be hypothesized that the assessment of diastolic dysfunction by TTE may be used to prescreen CCS patients before cMRI is performed to detect active myocardial inflammation. However, further studies are needed to evaluate this issue.

On average, LVEF was decreased in CSS patients as compared with controls, with 7 patients showing overt systolic heart failure $(\mathrm{EF}<50 \%)$. CSS patients with LVEF $<50 \%$ demonstrated decreased $\varepsilon$ cc, which may reflect the dysfunction of middle layer myocardial fibers. Chan et al reported that circumferential strain is decreased when transmurality of myocardial infarction exceeds $25 \% .{ }^{34}$ Consequently, it may be speculated that decreased LV systolic function results from involvement of not only the inner but also the middle layer of myocardial fibers. Interestingly, CSS patients with LVEF $\geq 50 \%$ had normal $\varepsilon_{\mathrm{cc}}$, suggesting preserved function of the middle layer myocardial fibers in the normally contracting LV. LV diastolic function was also impaired in CSS patients, a phenomenon already observed by Pela et al. ${ }^{12}$ Diastolic dysfunction accompanied systolic in 6 of 7 patients, and appeared as an isolated finding in 2. Diastolic dysfunction may be related to increased myocardial stiffness caused by both ongoing myocardial inflammation and/or myocardial fibrotic lesions. Valve abnormalities were mild and not common ( $20 \%$ of patients), again in agreement with Pela et al, but at variance with an earlier report, ${ }^{11}$ a contradiction probably explained by more aggressive current CSS treatment strategies.

ECG stress test (with normal epicardial coronary arteries) was positive in 4 patients with CSS and in none of the controls. The prevalence of a positive stress test might have been higher if 2 of the patients had not been excluded from the examination (both of them with symptomatic heart failure). Stress test ECG changes in CSS patients are probably explained by the infiltration of small myocardial vessels by eosinophils and/or vasculitis. Involvement of the epicardial coronary arteries in CSS seems rare. ${ }^{35,36}$ In the past, 4 patients in this group suffered from angina symptoms and/or elevated cardiac enzymes during exacerbation of their disease, but the epicardial coronary vessels on coronary angiography looked normal.

ANCA were present in $25 \%$ of the patients, lower than in a previous study, ${ }^{3}$ perhaps because the antibodies were not tested in all patients prior to the initiation of corticosteroid treatment. Generally, however, in CSS patients ANCA correlate negatively with heart involvement. ${ }^{3,8}$ In this group we did not observe differences when ANCA were taken to account. Peak eosinophilia, measured before initiation of corticosteroid treatment, showed higher values in patients with systolic heart failure and rhythm disturbances than in those with normal LV function and was negatively associated with EF, which is in agreement with previous reports ${ }^{15,37}$ and points to the possible relevance of eosinophilia in the pathogenesis of heart abnormalities in CSS. The role of vasculitis is less clear, but can not be ruled out. Other laboratory parameters measured, including NT-proBNP, were not useful in assessing the heart involvement in non-symptomatic CSS patients.

\section{Study Limitations}

The number of enrolled patients was relatively small and the results should be confirmed in a larger cohort. In the current study, no attempt was made to assess diastolic function by cMRI, although several cMRI methods for the evaluation of LV diastolic function are currently available. ${ }^{38}$ Finally, we did not perform serial follow-up examinations in patients with evidence of active inflammation and as such we could not evaluate the efficacy of immunosuppressive therapy on regression of myocardial edema. In the current study, tissue biopsy was not performed, which might have further elucidated the pathogenesis of the ongoing myocarditis.

In summary, heart involvement is highly prevalent among CSS patients in remission. At this stage most of these changes have a fibrotic, irreversible character. Nevertheless, in some patients active, inflammatory changes as assessed by cMRI are still present and in such patients more aggressive immunosuppression may be required, despite the criteria for disease remission beinge met. Our results also suggest that cMRI, providing the most comprehensive information, should be performed in all newly diagnosed CSS patients (especially those with highly elevated peripheral blood eosinophilia), even in the absence of cardiac symptoms, to detect early, potentially reversible changes. Immediate initiation of immunosuppressive treatment might limit irreversible myocardial damage.

\section{Acknowledgments}

The authors thank Dr Jessica Noelting and Dr George Zawadowski for their invaluable technical support.

\section{References}

1. Pagnoux C, Guilpain P, Guillevin L. Churg-Strauss syndrome. Curr Opin Rheumatol 2007; 19: 25-32.

2. Noth I, Strek ME, Leff AR. Churg-Strauss syndrome. Lancet 2003; 361: $587-594$

3. Sinico RA, Di Toma L, Maggiore U, Bottero P, Radice A, Tosoni C, et al. Prevalence and clinical significance of antineutrophil cytoplasmic antibodies in Churg-Strauss syndrome. Arthritis Rheum 2005; 52: $2926-2935$

4. Guillevin L, Cohen P, Gayraud M, Lhote F, Jarrousse B, Casassus P. Churg-Strauss syndrome: Clinical study and long-term follow-up of 96 patients. Medicine (Baltimore) 1999; 78: 26-37.

5. Knockaert DC. Cardiac involvement in systemic inflammatory diseases. Eur Heart J 2007; 28: 1797 - 1804.

6. Hellemans S, Dens J, Knockaert D. Coronary involvement in the Churg-Strauss syndrome. Heart 1997; 77: 576-578.

7. Pagnoux C, Guillevin L. Cardiac involvement in small and mediumsized vessel vasculitides. Lupus 2005; 14: 718-722.

8. Sable-Fourtassou R, Cohen P, Mahr A, Pagnoux C, Mouthon L, Jayne D, et al. Antineutrophil cytoplasmic antibodies and the Churg-Strauss syndrome. Ann Intern Med 2005; 143: 632-638.

9. Szczeklik W, Sokolowska B, Mastalerz L, Miszalski-Jamka T, Musial J. Heart involvement detected by magnetic resonance in a patient with Churg-Strauss syndrome, mimicking severe asthma exacerbation. Allergy 2010; 65: 1063-1064.

10. Setoguchi M, Okishige K, Sugiyama K, Shimura T, Maeda M, 
Aoyagi $\mathrm{H}$, et al. Sudden cardiac death associated with ChurgStrauss syndrome. Circ J 2009; 73: 2355-2359.

11. Morgan JM, Raposo L, Gibson DG. Cardiac involvement in ChurgStrauss syndrome shown by echocardiography. Br Heart J 1989; 62: $462-466$

12. Pela G, Tirabassi G, Pattoneri P, Pavone L, Garini G, Bruschi G. Cardiac involvement in the Churg-Strauss syndrome. Am J Cardiol 2006; 97: 1519-1524.

13. Baccouche H, Yilmaz A, Alscher D, Klingel K, Val-Bernal JF, Mahrholdt H. Images in cardiovascular medicine: Magnetic resonance assessment and therapy monitoring of cardiac involvement in Churg-Strauss syndrome. Circulation 2008; 117: 1745-1749.

14. Wassmuth R, Gobel U, Natusch A, Schneider W, Kettritz R, Dietz $\mathrm{R}$, et al. Cardiovascular magnetic resonance imaging detects cardiac involvement in Churg-Strauss syndrome. J Card Fail 2008; 14: $856-860$.

15. Neumann T, Manger B, Schmid M, Kroegel C, Hansch A, Kaiser WA, et al. Cardiac involvement in Churg-Strauss syndrome: Impact of endomyocarditis. Medicine (Baltimore) 2009; 88: 236-243.

16. Hansch A, Pfeil A, Rzanny R, Neumann T, Kaiser WA. First-pass myocardial perfusion abnormalities in Churg-Strauss syndrome with cardiac involvement. Int J Cardiovasc Imaging 2009; 25: 501-510.

17. Marmursztejn J, Vignaux O, Cohen P, Guilpain P, Pagnoux C, Gouya $\mathrm{H}$, et al. Impact of cardiac magnetic resonance imaging for assessment of Churg-Strauss syndrome: A cross-sectional study in 20 patients. Clin Exp Rheumatol 2009; 27(Suppl 52): S70-S76.

18. Dennert RM, van Paassen P, Schalla S, Kuznetsova T, Alzand BS, Staessen JA, et al. Cardiac involvement in Churg-Strauss syndrome. Arthritis Rheum 2010; 62: 627-634.

19. Masi AT, Hunder GG, Lie JT, Michel BA, Bloch DA, Arend WP, et al. The American College of Rheumatology 1990 criteria for the classification of Churg-Strauss syndrome (allergic granulomatosis and angiitis). Arthritis Rheum 1990; 33: 1094-1100.

20. Luqmani RA, Bacon PA, Moots RJ, Janssen BA, Pall A, Emery P, et al. Birmingham Vasculitis Activity Score (BVAS) in systemic necrotizing vasculitis. $Q J$ Med 1994; 87: 671-678.

21. Fletcher GF, Balady GJ, Amsterdam EA, Chaitman B, Eckel R, Fleg J, et al. Exercise standards for testing and training: A statement for healthcare professionals from the American Heart Association. Circulation 2001; 104: 1694-1740.

22. Hor KN, Gottliebson WM, Carson C, Wash E, Cnota J, Fleck R, et al. Comparison of magnetic resonance feature tracking for strain calculation with harmonic phase imaging analysis. JACC Cardiovasc Imaging 2010; 3: 144-151.

23. Friedrich MG, Strohm O, Schulz-Menger J, Marciniak H, Luft FC, Dietz R. Contrast media-enhanced magnetic resonance imaging visualizes myocardial changes in the course of viral myocarditis. Circulation 1998; 97: 1802-1809.

24. Nagueh SF, Appleton CP, Gillebert TC, Marino PN, Oh JK, Smiseth $\mathrm{OA}$, et al. Recommendations for the evaluation of left ventricular diastolic function by echocardiography. J Am Soc Echocardiogr 2009; 22: 107-133.

25. Zoghbi WA, Enriquez-Sarano M, Foster E, Grayburn PA, Kraft CD, Levine RA, et al. Recommendations for evaluation of the severity of native valvular regurgitation with two-dimensional and Doppler echocardiography. J Am Soc Echocardiogr 2003; 16: 777-802.

26. Friedrich MG, Sechtem U, Schulz-Menger J, Holmvang G, Alakija $\mathrm{P}$, Cooper LT, et al. Cardiovascular magnetic resonance in myocarditis: A JACC White Paper. J Am Coll Cardiol 2009; 53: 14751487.

27. Ishida M, Kato S, Sakuma H. Cardiac MRI in ischemic heart disease. Circ J 2009; 73: 1577-1588.

28. Kwon DH, Smedira NG, Rodriguez ER, Tan C, Setser R, Thamilarasan M, et al. Cardiac magnetic resonance detection of myocardial scarring in hypertrophic cardiomyopathy: Correlation with histopathology and prevalence of ventricular tachycardia. $J$ Am Coll Cardiol 2009; 54: 242-249.

29. Lin D, Kramer CM. Late gadolinium-enhanced cardiac magnetic resonance. Curr Cardiol Rep 2008; 10: 72-78.

30. Oka K, Tsujino T, Nakao S, Lee-Kawabata M, Ezumi A, Masai M, et al. Symptomatic ventricular tachyarrhythmia is associated with delayed gadolinium enhancement in cardiac magnetic resonance imaging and with elevated plasma brain natriuretic peptide level in hypertrophic cardiomyopathy. J Cardiol 2008; 52: 146-153.

31. Loeffler W. So-called endocarditis lenta. Sem Hop 1952; 28: $3693-$ 3699 (in Undetermined Language).

32. Val-Bernal JF, Mayorga M, Garcia-Alberdi E, Pozueta JA. ChurgStrauss syndrome and sudden cardiac death. Cardiovasc Pathol 2003; 12: 94-97.

33. Guillevin L, Lhote F, Gayraud M, Cohen P, Jarrousse B, Lortholary $\mathrm{O}$, et al. Prognostic factors in polyarteritis nodosa and Churg-Strauss syndrome: A prospective study in 342 patients. Medicine (Baltimore) 1996; 75: $17-28$.

34. Chan J, Hanekom L, Wong C, Leano R, Cho GY, Marwick TH. Differentiation of subendocardial and transmural infarction using two-dimensional strain rate imaging to assess short-axis and longaxis myocardial function. J Am Coll Cardiol 2006; 48: 2026-2033.

35. Kozak M, Gill EA, Green LS. The Churg-Strauss syndrome: A case report with angiographically documented coronary involvement and a review of the literature. Chest 1995; 107: 578-580.

36. Mavrogeni S, Manoussakis MN, Karagiorga TC, Douskou M, Panagiotakos D, Bournia V, et al. Detection of coronary artery lesions and myocardial necrosis by magnetic resonance in systemic necrotizing vasculitides. Arthritis Rheum 2009; 61: 1121-1129.

37. Churg J, Strauss L. Allergic granulomatosis, allergic angiitis, and periarteritis nodosa. Am J Pathol 1951; 27: 277-301.

38. Kawaji K, Codella NC, Prince MR, Chu CW, Shakoor A, LaBounty TM, et al. Automated segmentation of routine clinical cardiac magnetic resonance imaging for assessment of left ventricular diastolic dysfunction. Circ Cardiovasc Imaging 2009; 2: 476-484.

\section{Supplemental Files}

Supplemental File 1

Table S1. Patients' Characteristics Using a Case-Sensitive Approach

Please find supplemental file(s);

http://dx.doi.org/10.1253/circj.CJ-10-0772 